\title{
Keynote-puheenvuoro
}

Prologi - puheviestinnän vuosikirja 2017

60-65

\section{Rethinking virtuality in a digital media age}

\author{
Jennifer L. Gibbs
}

Professor

Department of Communication

University of California, Santa Barbara

gibbs@comm.ucsb.edu

\begin{abstract}
Scholars have studied virtuality in teams and organizations for over two decades. The term "virtual" is often used loosely and imprecisely, and theoretical debates have flourished over what differentiates virtual from non-virtual teams. In these debates, scholarship has not explicitly considered the significant ways in which the technological landscape has changed over this time. While the virtual is often treated as a separate space from "real", physical or face-to-face interaction, the increasing technological saturation of our lives has resulted in a blurring of online and offline worlds such that these distinctions may no longer hold up. I will explore whether the term "virtuality" still has currency and the ways in which we must rethink our underlying assumptions about virtuality in a digital media age.
\end{abstract}




\section{Introduction}

Scholars have been studying virtuality for over two decades, since I was in graduate school. The term "virtual" has primarily been associated with online contexts such as virtual teams, virtual communities, virtual worlds, and virtual reality. The meaning of the term has, however, always been contested, and its standing precarious. It is often used interchangeably with related terms such as distributed, computer-mediated, online, or remote. It generally refers to separation in space and time and reliance on digital technologies. The term "virtual" is often used loosely and imprecisely, however. This is most evident in the virtual teams literature, where debates have flourished over what differentiates virtual from non-virtual teams, and which dimensions to include in conceptualizations of virtuality.

One point that is rarely explicitly considered, however, is the significant ways in which the technological landscape has changed over the past two decades: our lives have become technologically saturated. Early research often focused on purely virtual (or computer-mediated) versus purely collocated or face-to-face settings, regarding online and offline environments as separate spaces. There has been a growing recognition that online and offline worlds are not separate but are intertwined, such that these distinctions have become blurred and potentially meaningless. While early conceptualizations regarded the virtual as a separate space from "real", face-to-face interaction, this may no longer be the case now that new communication technologies are increasingly incorporated into our personal and work lives. This changing technological landscape requires a rethinking of what virtuality means, and whether and how we need to rethink our assumptions about virtuality in communication scholarship. In this talk,
I wish to pose the following questions: Has the term "virtual" become meaningless? How should we rethink virtuality in a digital media age?

\section{The etymology and ideology of virtual}

Nohria and Berkley (1994) trace the history of the term "virtual" back to at least the mid19th century in the physical sciences, where it was used to refer to "structures and objects whose ontological status lies in the fuzzy realm between fact and apparition" (p. 113) such as a virtual image, in which light appears to emanate but does not actually do so. Since the late 1980s, the term virtual has taken on currency in describing computerized technologies that provide simulations of physical reality. Implicit in these views is an assumption that the "virtual" is a separate space that is divorced from reality - which is assumed to be grounded in face-toface interactions. Early views conjured up images of cyborgs, artificial intelligence, and virtual reality, which were often imbued with utopian or dystopian views about new technologies and their often exaggerated role in helping or hindering society. Our societal exploration of the implications of new technologies is often seen in science fiction and popular culture images of technology taking over our lives, extending our capabilities yet with sometimes disastrous consequences (e.g., movies and shows such as Minority Report, The Matrix, Her, and Black Mirror). Scholarship on virtuality often subconsciously draws on these views, regarding virtuality either as a boon or a bane.

\section{Virtuality as boon}

Virtual organizations have been conceptualized as having networked, flattened structures, and as being more ephemeral and permeable. These characteristics have led managers and prac- 
titioners to extol the virtues of virtuality in affording round-the-clock work with little or no infrastructure, overhead, or costs. Much of the early managerial discourse on the virtual workplace tended to celebrate virtuality - defined in terms of technological infrastructure - as the key to success in an increasingly competitive global marketplace. In this discourse, the "virtual" metaphor connoted the benefits of streamlined efficiency, flexibility, and ease of operating without the baggage of high overhead costs, cumbersome bureaucratic structures, and the human resources difficulties of managing actual people. By extension, the virtual organization, fueled by an infrastructure of advanced information technology, should "virtually" run itself. Such views tended to focus attention on the potential capabilities of state-of-the-art technologies and away from the social challenges of organizing that may not be stripped away by the virtual structure but may in fact become even more critical. This is evident in notions such as the "organizationless organization" (Davidow \& Malone, 1992) or the "boundaryless organization” (Ulrich, Jick, Kerr, Prahalad, \& Bossidy, 2002). Despite the promise of technological liberation from the shackles of structure and hierarchy, this view has not been borne out in research on virtual teams and organizations.

\section{Virtuality as bane}

While the utopian rhetoric of the virtual organization has been largely unsubstantiated, the growing body of research on virtual teams has painted a bleaker view of virtuality as fraught with challenges. Early research on virtual teams tended to set up experiments that contrasted purely computer-mediated (virtual) teams with purely face-to-face teams, in which faceto-face teams were generally found to out-perform virtual teams. Reanalyses of these studies found that computer-mediated groups perfor- med as well as face-to-face groups when given more time for relationship formation and that the reduced social cues in computer-mediated settings could actually lead to idealized impressions and more intimate relationships. However, the virtual teams literature remains dominated by cues-filtered-out approaches (Walther \& Parks, 2002), which assume that computer-mediated - or virtual - communication is deficient in promoting interpersonal relationships because of its reduced nonverbal and social cues, and implicitly compares virtual to face-to-face teams as the gold standard. Much of this literature relies on a "deficiency" view of virtuality that focuses on the interpersonal challenges that arise due to reduced social cues (Gibbs, Nekrassova, Grushina, \& Abdul Wahab, 2008).

\section{Rethinking virtuality}

Scholarship has made several key advances in conceptualizing virtuality. First, we have moved away from notions of virtuality as an "on-off switch" (e.g., teams are either virtual or non-virtual) to viewing virtuality as a continuum on which teams can be arrayed from low to high. Second, there is general consensus that virtuality is a multidimensional construct. While there is still debate over which dimensions to include, key dimensions that I have used in my research are geographical dispersion, electronic dependence, cultural diversity, and dynamic structure (Gibson \& Gibbs, 2006). Third, scholars have begun to regard virtuality as subjectively experienced rather than based on objective structural characteristics such as number of locations or time zones crossed. Finally, rather than seeing virtual teams as a unique breed of team, scholars are starting to recognize virtuality as a ubiquitous feature of modern teams (to the extent that nearly all teams now use technology and thus are at least somewhat virtual). 
As debates about the promise and perils of virtuality have played out over the past two decades, the technology landscape has changed significantly. Our relationship with technology as well as the tools we have available have changed significantly. While early research on virtuality focused on leaner technologies such as computer conferencing, group decision support systems, and email, new tools such as social media are richer and more interactive, and the portability of smart phones and other mobile devices has resulted in them becoming more integrated in our lives as we carry them around with us. This has led to a shift in the role digital media technologies play in our lives such that online and offline interactions have become more and more blurred (rather than separate worlds in which online is distinguished from "real" interaction), and they have become deeply interwoven with one another and almost inseparable. Evidence is amassing that rather than supplanting sources of offline community, new technologies supplement and extend our everyday and work interactions in meaningful ways.

Our transformed relationship with technology requires new ways of thinking about virtuality. This begs the question of whether the term "virtuality" holds currency any longer in a social media age in which distinctions between "online" and "offline" have become obsolete. Does it make sense to talk about virtual teams, organizations, or communities any longer? Given that it is rare to find completely collocated teams that do not use any technologies to collaborate (just as it is rare to find teams that have no face-to-face interaction), we need to rethink our notions about virtuality and the extent to which it even makes sense to talk about virtual teams anymore - given that increasingly all teams and organizations are, to varying extents, virtual. I argue that we need to rethink and col- lapse dualisms: unreal vs. real, online vs. offline, virtual vs. physical.

\section{From disconnection to constant connection}

Along with the changing technological landscape has come a shifting set of concerns around virtuality. While the dominant concern among scholars of virtuality has been one of disconnection - in other words, helping virtual workers to become less isolated from behind computer screens and become more connected with one another - with mobile digital media has come a new set of concerns, centering around excessive or constant connection. Whereas early virtual teams research emphasized the disconnection of members due to their physical dispersion and separation by technology and struggled to find ways to connect them, the literature has shifted to emphasize the drawbacks of excessive connection among virtual workers who are both able and expected to be continually connected to work through the electronic tether of mobile devices. Now that employees have the ability to attend to multiple demands beyond the normal workday and office through digital media that allow them to respond anytime, from anywhere, this is creating new expectations that they will be always available.

This new concern can be seen in concepts such as constant connectivity, perpetual contact, pervasive awareness, and digital ubiquity. It creates drawbacks of increased stress, cognitive load, and demands on one's time and attention. These concerns are evident in news headlines such as the following: "Stressed, Tired, Rushed: A Portrait of the Modern Family" and "Why Companies Fail to Engage Today's Workforce: The Overwhelmed Employee". One survey of working adults found that workers face new pressures to be always-on: two thirds (65\%) felt 
pressure to check their work email after hours, $55 \%$ reported having a hard time detaching from work, and 20\% reported high levels of emotional exhaustion (Belkin, Becker, \& Conroy, 2016).

In my new book, Distracted: Staying Connected Without Losing Focus (Kurtzberg \& Gibbs, 2017), I examine these concerns. My co-author and I pose the following questions: Are we more distracted today than in the past? Are digital devices making us more distracted? We argue that we are living in an age of distraction. It is important to note that distraction is not new; it has always been part of the human condition. For example, Buddhist practices of meditation were invented to calm the chattering "monkey mind" and restore mindfulness. Distraction also has psychological value when it comes in the form of regenerative breaks or downtime. In fact, the excessive busyness we face due to our culture of overwork today may make our need for distraction even greater. While our motivations for distraction have not changed, we now have new tools of distraction. Digital media contribute new sources of distraction and interruption. Mobile phone users check their phones an average of 150 times per day. Knowledge workers average only a few minutes on a task before switching tasks. Our use of multiple media has contributed to the fragmentation of our work experience, such that multi-tasking has become the new normal.

The pressures of constant connectivity and their resulting distractions and interruptions have led to communicative efforts by workers to disconnect or disengage from the demands of new technologies on their time and attention. In one study of distributed engineers in a high-tech start-up, my co-authors and I found that the excessive openness of social media tools created tensions for these virtual wor- kers between visibility vs. invisibility, engagement vs. disengagement, and sharing vs. control. They managed these tensions strategically through covert efforts to limit their visibility, engagement, and knowledge sharing in order to manage demands on their time and attention (Gibbs, Rozaidi, \& Eisenberg, 2013). This is one of a number of studies that suggests that, rather than artificially separating us from our real, offline lives, the increased role of technologies in our daily personal and work lives has enhanced our connections and intensified our interactions - sometimes too much.

So what is the way forward? I argue that our assumption that the "virtual" occupies a separate, technologically-mediated world that is divorced from the real, physical, face-to-face world is no longer tenable (if it ever was). Scholarship has shifted to recognize that online interactions are just as "real" as offline interactions, and that the two are intertwined and increasingly inseparable. Dichotomies between unreal vs. real, online vs. offline, and virtual vs. physical no longer hold up in today's increasingly technologically saturated world, although these outdated assumptions continue to undergird much of our research. Virtuality still matters, but it may be moving from the foreground to the background, from figure to ground. It has become so ubiquitous that we are no longer attuned to it or primed to focus on it. We no longer have virtual teams and virtual workers, just teams and workers. This has led to us to a different set of concerns. Our concerns have shifted from how to connect virtual workers to how to alleviate their excessive connection. We need to better account for the tensions between autonomy and connectivity in (virtual) work. Virtual workers are now more connected to one another through technology that allows them to work across time and space. But boundaries are not erased, nor are bureaucracy or structure 
eliminated. Virtuality requires engaging with difference and complexity and provides new structures for doing so. This process will only become more important as digital media become infused into the workplace.

\section{References}

Belkin, L. Y., Becker W. J., \& Conroy, S. A. (2016). Exhausted, but unable to disconnect: After-hours email, work-family balance and identification. Academy of Management Proceedings, January 2016.

Davidow, W. H., \& Malone, M. S. (1992). The virtual corporation. New York: Harper Business.

Gibbs, J. L., Nekrassova, D., Grushina, Y., \& Abdul Wahab, S. (2008).

Reconceptualizing virtual teaming from a constitutive perspective: Review, redirection, and research agenda. In C. S. Beck (Ed.), Communication yearbook 32 (pp. 187-229). New York: Routledge.

Gibbs, J. L., Rozaidi, N. A., \& Eisenberg, J. (2013). Overcoming the "ideology of openness": Probing the affordances of social media for organizational knowledge sharing. Journal of Computer-Mediated Communication, 19(1), 102-120.

Gibson, C. B., \& Gibbs, J. L. (2006). Unpacking the concept of virtuality: The effects of geographic dispersion, electronic dependence, dynamic structure, and national diversity on team innovation. Administrative Science Quarterly, 51, 451-495.

Kurtzberg, T., \& Gibbs, J. L. (2017). Distracted: Staying connected without losing focus. Santa Barbara, CA: Praeger Press.

Nohria, N., \& Berkley, J. D. (1994). The virtual organization: Bureaucracy, technology, and the implosion of control. In C. Heckscher \& A. Donnellon (Eds.), The post-bureaucratic organization: New perspectives on organizational change (pp. 108-128). Thousand Oaks, CA: Sage.

Ulrich, D., Jick, T., Kerr, S., Prahalad, C. K., \& Bossidy, L. A. (2002). The boundaryless organization: Breaking the chains of organizational structure. San Francisco, CA: Jossey-Bass.
Walther, J. B., \& Parks, M. R. (2002). Cues filtered out, cues filtered in: Computer-mediated communication and relationships. In M. L. Knapp \& J. A. Daly (Eds.). Handbook of interpersonal communication (3rd ed., pp. 529-563). Thousand Oaks, CA: Sage. 